\title{
Static response in disk packings
}

\author{
Cristian F. Moukarzel, Hector Pacheco-Martínez, J. C. Ruiz-Suarez \\ CINVESTAV del IPN, Depto. de Física Aplicada, \\ 97310 Mérida, Yucatán, México \\ Ana M. Vidales \\ Depto. de Física y CONICET, Universidad Nacional de San Luis \\ Chacabuco 917, 5700 San Luis, San Luis, Argentina
}

(Dated: October 3, 2018)

\begin{abstract}
We present experimental and numerical results for displacement response functions in packings of rigid frictional disks under gravity. The central disk on the bottom layer is shifted upwards by a small amount, and the motions of disks above it define the displacement response. Disk motions are measured with the help of a still digital camera. The responses so measured provide information on the force-force response, that is, the excess force at the bottom produced by a small overload in the bulk. We find that, in experiments, the vertical-force response shows a Gaussianlike shape, broadening roughly as the square root of distance, as predicted by diffusive theories for stress propagation in granulates. However, the diffusion coefficient obtained from a fit of the response width is ten times larger than predicted by such theories. Moreover we notice that our data is compatible with a crossover to linear broadening at large scales. In numerical simulations on similar systems (but without friction), on the other hand, a double-peaked response is found, indicating wave-like propagation of stresses. We discuss the main reasons for the different behaviors of experimental and model systems, and compare our findings with previous works.
\end{abstract}

PACS numbers: 45.70.-n, 45.70.Cc, 83.80.Fg

\section{INTRODUCTION}

Stress distributions in static granular materials display puzzling characteristics [1, 2, 3, 4] that do not quite fit into classical elastic descriptions, and have defied attempts at analytic modeling for some time already. The observation of a pressure dip below conical piles, force chains, sudden macroscopic changes in stress patterns under slight perturbations, and exponential (instead of Gaussian) stress distributions, among other phenomena, have triggered intense theoretical and experimental work. As a result, a multiplicity of stress propagation models have been put forward. The $q$-model [5, [6] assumes diffusive behavior for the vertical stress component considered as a scalar quantity, and gives rise to an exponential distribution of stresses. Other scalar models in turn predict Gaussian [7] or power-law distributed [8] stresses. By postulating a linear relation between stress components [9], a wavelike equation [10] is derived for stress propagation, the so called OSL model 11]. This model reproduces the pressure dip [10], and is consistent with stresses in silos 12]. A memory formalism 13, 14 contains as special limits the wavelike and diffusive behaviors. Furthermore, a recent description in terms of scattering force-chains [15] gives rise to wavelike propagation on small scales, crossing over to something similar to classical elasticity on larger scales.

Linear elasticity describes the propagation of stresses in terms of differential equations of the elliptic type, wavelike propagation corresponds to the hyperbolic case, while diffusive behavior is the borderline, or parabolic case. These three descriptions give rise to very different responses [3, 4] when a small force is applied on a localized region on the upper surface of a packing. Linear elasticity predicts a bell-shaped response, having a width proportional to depth. A diffusive behavior, on the other hand, implies that the width of the response scales as the square root of depth. Finally, a wavelike propagation would be evidenced by a response that is maximum on a diffuse annulus of linearly growing radius (the "light-cone") in three dimensions, or by a response showing two diverging peaks in two dimensions.

One might expect such differences to be easily resolved by properly designed experiments. However, presently available experimental results are not conclusive. Some small-scale results support the validity of the $q$ model [16], while recent experiments using photoelastic techniques 17] appear to be in conflict with a diffusive picture. The memory formalism has been shown to reproduce the stress oscillations observed in laterally confined packings [18]. Experiments on sand [19, 20] show a single-peaked response function whose width scales linearly with depth, as predicted by elasticity. However the precise shape of the response does not quite match 20] that of a linear elastic medium. It has been noted that even systems in the elliptic regime can have two peaks in their response functions 21]. It appears at present difficult, based on available experimental results, to clearly validate, or disprove, any of the stress propagation models that have been proposed.

Numerical measurements on disordered packings of frictionless disks, that respect the property of isostaticity [22, 23], both on-lattice 24, 25] (with contact disorder) and off-lattice [26, 27, 28], show two clearly distinguishable peaks in their response functions. How- 
ever in experiments wavelike (or hyperbolic) response functions have only been observed in ordered packings [29], and it has been argued that disorder produces a crossover to an elliptic description on large scales.

Notice that the measurement of response functions in granular packings poses a subtle experimental challenge. A distinction must be made between the response to infinitesimal perturbations $G^{i}$, derived under the assumption that the contact network does not change, and $G^{f}$, the response under small but finite perturbations, i.e. allowing for rearrangements. On disordered isostatic packings, $G^{i}$ is singular [22, 24, 25] and does not have a well defined continuum limit. This is so because the propagation of stresses is described by random multiplicative processes on these systems. In practice this implies that $G^{i}$ takes positive as well as negative values, whose modulus grows exponentially with distance from the point where the perturbation is applied. Thus on isostatic disordered packings, any finite perturbation, no matter how small, necessarily induces contact rearrangements, because a large and negative Green function corresponds to a contact that will open upon perturbation. This anomalous sensitivity to perturbation is due to isostaticity, and has been suggested as being responsible for the tendency of stiff packings to reorganize upon perturbation [22]. Based on very general physical grounds, one can expect rearrangements to fundamentally modify response functions. Thus one should expect experimentally measured response functions to strongly depend on the magnitude of the perturbation whenever isostaticity is satisfied. Possible effects of rearrangements are discussed for example in Ref. [23] (See also recent discussions in Refs. 30, 31]). Moreover, isostaticity has only been rigorously proven [22, 23] for frictionless packings. It is known that friction gives rise to indeterminacies [32, 33], and this is not compatible with isostaticity. In real packings friction is important, and it is at present not clear whether isostaticity applies in some restricted sense, or not at all. Recent molecular dynamics results 34] on frictional deformable spheres are not compatible with the packing being isostatic, although previous similar studies [35] reached different conclusions.

Interparticle forces in granular packings have been previously measured by means of carbon-paper experiments 1, 5, 36, 37, 38], photoelastic techniques [17, 29], pressure sensors [19, 20], and high-precision balances [39]. None of these methods is optimal for the determination of response functions, a task that requires the measurement of small forces over small regions. Several of the methods used up to now require large forces to be applied to the packing, while others are not able to detect forces on single particles but only averages over relatively large regions. The use of an electronic balance gives very precise results, but requires an extremely time consuming scanning of the bottom of the packing.

In this paper we report our first results from a novel experimental technique allowing precise measurements of response functions in two-dimensional packings. Our procedure consists in measuring the displacementdisplacement response function [22, 24, 25], that is, the displacement produced on a given disk by an upward displacement of one of the disks on the lowermost layer. For isostatic systems, it has been shown [22, 24, 25] that this quantity is exactly equivalent to the stressstress response function. Several recent numerical studies make use of this equivalence to measure stress responses [22, 24, 25, 26, 27, 28], however this is the first time that this equivalence between stress-stress and displacement-displacement response functions is used in experiments.

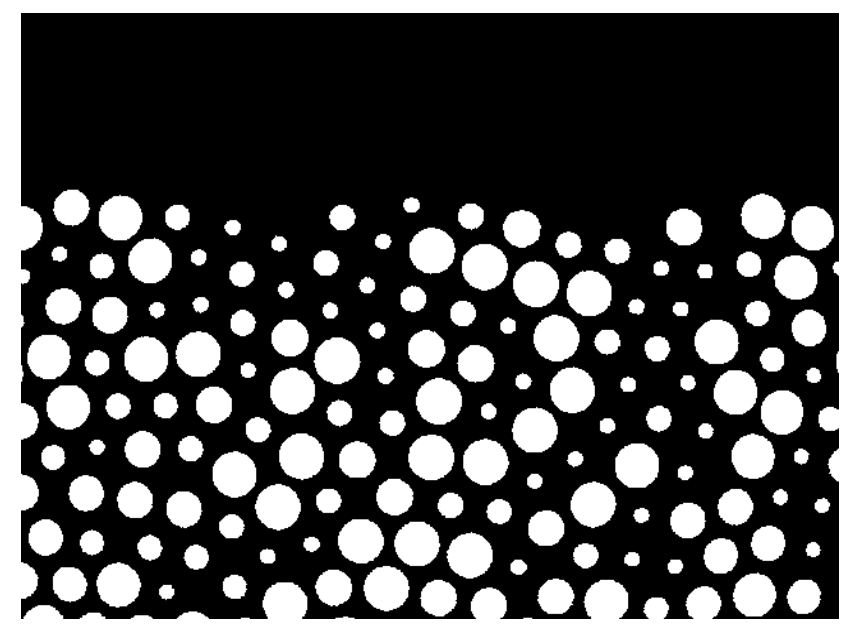

FIG. 1: A disk configuration as seen by the camera. The camera resolution is $640 \times 480$ pixels. The approximate size of the viewable field is $29 \times 22 \mathrm{~cm}$, implying a spatial resolution of roughly 0.45 millimeter per pixel. Only around 150 disks out of a total of 400 in the container, fit in the camera viewfield. The average number of layers is between nine and ten.

In standard response function measurements, a small force $f$ is applied on a point on the top surface of a packing (defined as the origin of coordinates) and the response function $G(x, y)$ is defined as the excess stress induced at $\{x, y\}$, divided by $f$. In our experiments, the response function $G(x, y)$ gives the excess stress at $\{0,0\}$ (the central particle on the lowermost row of the packings) produced by a small force $f$ acting at $\{x, y\}$. If boundary effects can be neglected, these two definitions of $G(x, y)$ should give statistically equivalent results. In other words, although for a given sample these two ways to measure the response give different results, after sample averaging one obtains the same function, if translation invariance applies.

Some advantages of the experimental procedure presented in this work are as follows. The measurement of displacements can be done with much better precision and by simpler means than that of forces. Vertical and horizontal displacements provide information respectively on vertical $\left(G_{y}\right)$ and horizontal $\left(G_{x}\right)$ responses. 
We obtain information on $G_{x}(x, y)$ and $G_{y}(x, y)$ for all $\{x, y\}$ in one measurement. Moreover, our technique does not involve the subtraction of two force patterns, a procedure which is prone to error, and more so when two large force patterns are subtracted to obtain a small response. In our experiments, the displacement of each particle is a direct measurement of the green function at that point.

This work is organized in the following manner: Section IIA contains a description of the experimental setup used for the measurement of response functions. In Section $\amalg B$ our results are presented and analyzed. A comparison with numerical results obtained on frictionless packings is established in Section IIII Finally, our results are summarized and discussed in Section IV

\section{EXPERIMENTAL SETUP}

\section{A. Experimental device}

The experimental device consisted of a rectangular container made of two parallel $615 \mathrm{~mm}$ by $320 \mathrm{~mm}$ plexiglass plates. The back and front plates were respectively 34 and $10 \mathrm{~mm}$ thick, and they were separated by 5 mm. 400 aluminum disks with a thickness of $4 \mathrm{~mm}$ were confined between the plates. These disks had diameters $16,17,18$ and $19 \mathrm{~mm}$ (100 of each). The container was placed vertically in order to minimize friction effects between disks and walls. All disks were painted black, and a white circular label was affixed onto each of them in order to allow for motion measurement using digital means. Labels had a slightly smaller radius than the disks they were fixed on.

The disks' positions and movements were recorded using a still digital camera with a resolution of $640 \times 480$ pixels. The camera pointed to a rectangle of $29 \times 22 \mathrm{~cm}$ around the middle of the plexiglass container, implying a resolution of roughly $0.45 \mathrm{~mm}$ per pixel. Before each experiment $i$, the packing was shaken and allowed to settle under gravity alone. The disk configuration before perturbation was then recorded using the camera, obtaining a pre-image $I^{0}(i)$. Fig. 1 1 shows a typical image. Next the central disk in the lowest row was displaced upwards by $1 \mathrm{~mm}$. This disk was fixed to a micrometric screw that fitted through a specially devised hole in the bottom border of the container. At this point the final configuration of disks was recorded and stored to post-image $I^{f}(i)$. This procedure was repeated a total of 330 times.

A $C$ program processed these images to obtain individual disk motions. Our program took pairs of images (before and after the perturbation) in b\&w (1bpp) format as input. For each pre-image, all clusters of white pixels were first identified by the method of burning. Their geometric centers were taken to be disks centers. These centers were then taken as starting points for the identification (burning) of clusters on the corresponding postimage. This allowed to find the displacement suffered by each disk in the pre-image. For each disk, horizontal and vertical displacements $G_{x}$ and $G_{y}$ were calculated from the differences between geometric centers. These numbers (two per disk) give the displacement-displacement response function for a given packing, which for frictionless systems equals the force-force response functions, as discussed somewhere else [22, 24, 25]. Because of the existence of friction in our experiments, displacement responses are not exactly equal to stress responses, however these differences will be regarded as small and ignored in the following.

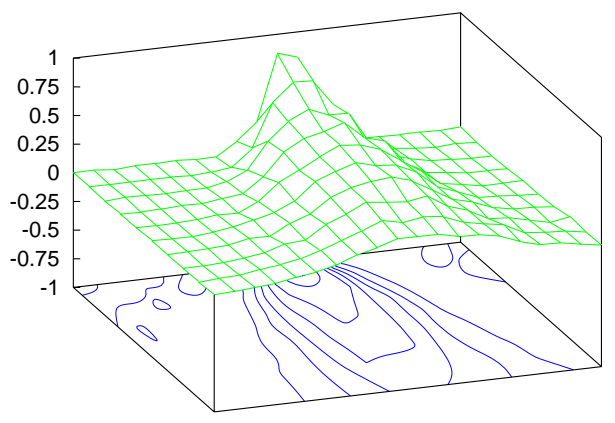

a)

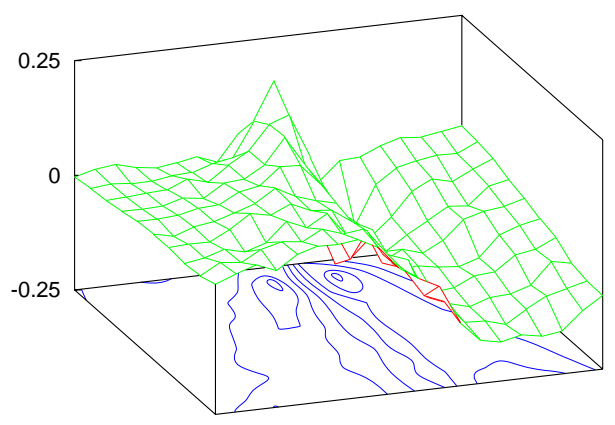

b)

FIG. 2: Response functions experimentally obtained after averaging over 330 packings of 400 disks. The displacement displacement response measures the displacement ((a) vertical, (b) horizontal) of a disk at $\{x, y\}$ in the bulk, produced by the upwards motion of a disk at $\{0,0\}$ in the lowermost layer. On isostatic systems this is exactly equal to the the the vertical excess force on the lowermost central grain at $\{0,0\}$ when a (respectively vertical or horizontal) unit force is applied at $\{x, y\}$.

Response functions were averaged over 330 repetitions of the experiment. In order to take averages we subdivide the image into square cells, adding values of $G$ only to the cell to which the center of the corresponding disk belongs before the displacement. 


\section{B. Experimental results}

Fig. 2a,b show our main results, respectively vertical and horizontal response functions. The horizontal response $G_{x}$ equals minus the excess compressive force at the bottom produced by a unit force acting in the positive $x$ direction in the bulk. Fig. $2 \mathrm{~b}$ indicates that, on average, compressive forces on the bottom increase on the right side of the load's application point, and decrease to the left of it. The $q$-model has no prediction for this quantity, as it only handles the vertical component of the force. It would be interesting to compare these results with the predictions of other competing stress-transmission theories.

As Fig. 3a shows, the vertical response $G_{y}$ has the form of a bell-shaped curve with a single peak, whose width grows with the distance from the perturbation point. Two models that predict a single-peaked response are the $q$-model [5, 6, 16], and classical elasticity. Within linear elasticity, the width $\omega(y)$ of the response grows linearly with distance, while in diffusive models like the $q$-model it grows with the square root of distance.

The width at half-height $\omega(y)$ of the vertical response function $G_{y}$ can be calculated from the data in Fig. 2 a. Our results are displayed in Fig. 3]. Taking $a, b, c$ as free parameters we fit $\omega(y)=a * y^{b}+c$ and obtain $b=0.51 \pm 0.08$. This result, taken at face value, supports diffuse behavior of stresses. Assuming diffusive behavior $(b=1 / 2)$ and fitting $w(y)=(2 D y)^{1 / 2}+c$, we obtain $D=95 \pm 5 \mathrm{~mm}$. This last result is not in very good agreement with the diffusive $q$-model theory, which predicts [16] $D \approx$ grain size/2. Given that we have equal numbers of disks of size 16,17, 18 and $19 \mathrm{~mm}$, the average size is $\approx 17.5 \mathrm{~mm}$. Thus we find $D \approx 5 \times$ grain size, a factor of 10 of from the theoretical prediction. With this evidence, we believe that a parabolic per se fit cannot be taken as strong evidence in favor of diffusive behavior at large scales, given the small number of layers (around 10) that we have in this experiment [4]. Notice that an asymptotically linear behavior of $\omega(y)$ is also consistent with our data (dotted line in 3), if deviations in the first few layers are ignored. Our preliminary conclusion is then that parabolic widening holds on very short scales, however with a possible crossover to linear widening on larger scales.

In Ref. [16] it is argued that an isostatic system of frictionless disks should behave according to the predictions of the $q$-model, i.e. diffusively. This expectation is not verified in simulations 22, 24, 25] in which the polydispersity is small and all disk centers are located on the sites of a triangular lattice (notice that, although disk centers are on a regular lattice, these systems have strong contact disorder). For these (on-lattice) isostatic systems, the average response function of frictionless hard disks shows two peaks that diverge linearly. This behavior is consistent with theoretical arguments 26] suggesting that the assumptions leading to wave-like propagation [9] of stresses are exact on isostatic [22] packings.

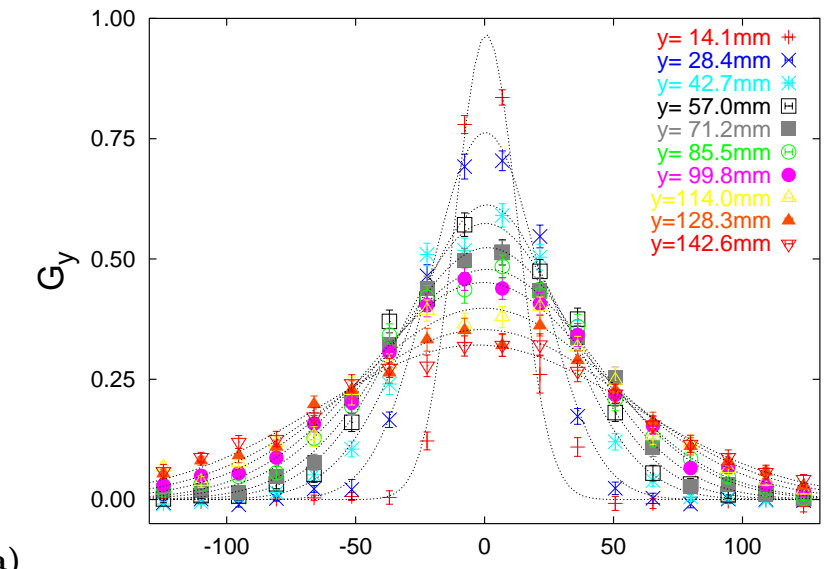

a)

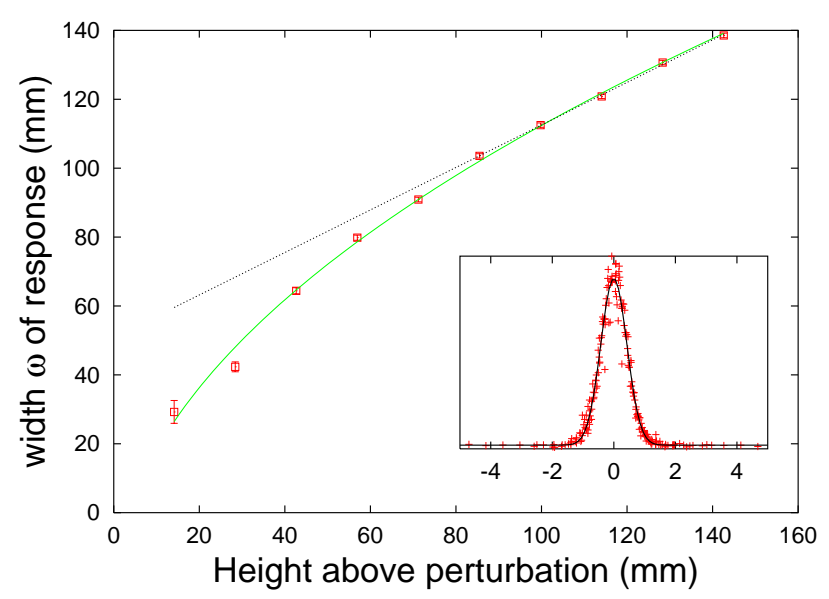

FIG. 3: a) Experimentally measured vertical response function $G_{y}$ at different heights $y$ above the bottom. The dashed lines are Gaussian fits. b) Width $\omega$ of $G_{y}(x, y)$ vs height $y$ above the perturbation point (squares). The solid line is a parabolic fit $w(y)=(2 D y)^{1 / 2}+c$ resulting in $D=95 \pm 5$ $\mathrm{mm}$. The dotted straight line has slope 0.62 , and fits the last five points. The inset shows the rescaled response function $\hat{G}=\omega G(x / \omega, y)$, for all values of $y$. The solid line is a Gaussian fit.

However it has been argued that the response might become single-peaked when the disorder is large [29]. In order to explore the effect of disorder in the positions of the disks, and for the sake of comparison with our own experiments, we performed numerical simulations to measure the response on systems of frictionless disks with the same distribution of radii as in the experiments. In these simulations, disks do not occupy the sites of a regular lattice. This is the subject of next section.

\section{NUMERICAL EXPERIMENTS}

The numerical experiments start by pouring disks, one by one, into a rectangular die, following a steepest descent algorithm [40, 41]. The equilibrium position for each grain is attained when its center of mass falls between the centers of two already deposited grains. This 
way of packing originates a sequentially deposited isostatic structure, however not necessarily a stable one (positive stresses) as is the case for the on-lattice simulations of Refs. 22, 24, 25], or the adaptive simulations in Refs. 27, 28, 31]. The geometric parameters (size and number of disks, size of the container, etc.) used in the simulations reproduce the scale of the real experiment previously discussed. There is no friction in our simulations.

Once the assembly is ready, the central particle at the bottom of the die is displaced upwards. Upon perturbing the system we do not allow for rearrangements, that is, we keep the list of contacts unchanged. The isostaticity of the contact network then allows one to calculate the displacement of all other particles very straightforwardly by upwards propagation [22, 24, 25, 41]. Because of the conservation of contacts our results are relevant for the limit of very small perturbations in frictionless systems. In experiments, rearrangements are very difficult to avoid when perturbing the system as we do, unless displacements are exceedingly small.

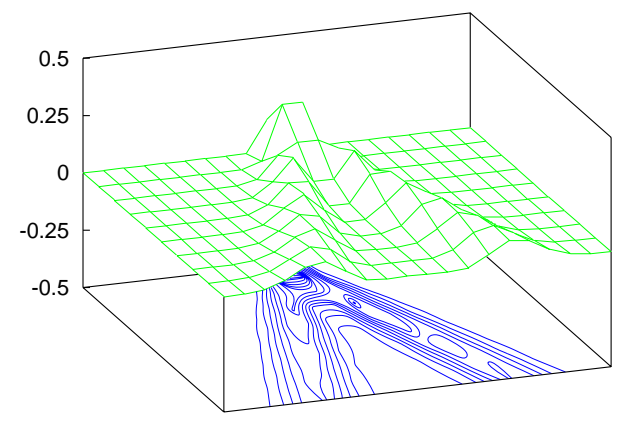

a)

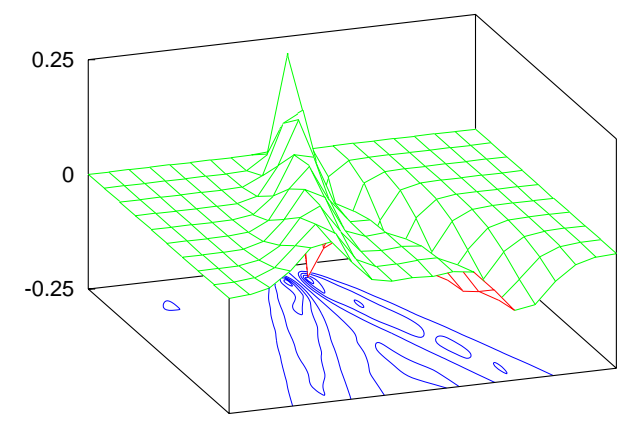

b)

FIG. 4: Numerical results for the vertical (a)) and horizontal (b)) response function, on model frictionless packings with the same geometry as in experiments. These responses are calculated assuming the limit of infinitesimally small perturbation, in which contacts between disks do not change.

Because of the existence of multiplicative effects 25] in the simulated experiment, we find the expected large fluc- tuations in the measured response functions, thus averages are taken over $10^{7}$ samples. Fig. 4 shows the results for the response functions, as measured in our simulations. It is clear that, also in this case as for regular packings [22, 24, 25] $G_{y}$ presents a double-peaked shape, characteristic of a hyperbolic 9, 28, 42] behavior. Thus, the amount of disorder considered in this work does not produce a single-peaked response in isostatic systems, although the equivalent but frictional experimental system (Fig. 2) displays a single peak. Comparable results are found with a more elaborate but time-consuming adaptive algorithm [27, 28, 31].

\section{DISCUSSION}

We have measured displacement response functions, both experimentally on arrays of frictional disks, and numerically for disks without friction. Because of the virtual work principle, for isostatic systems the displacement response equals the stress response. Our simulations do have this exact symmetry, however in experiments the existence of friction makes the system not isostatic in general [34]. Thus the displacement response is not necessarily equal to the stress green function, however we expect the differences between them to be small. In this work we have then assumed that stress responses take similar values to what we find experimentally for displacement responses. The experimentally measured response (Figs. 2] and 3) has a Gaussian-like shape, and its width scales approximately as the square root of the depth. This appears as consistent with predictions of the $q$-model 5, 6, 16]. However, this model predicts a diffusion coefficient $D$ whose value is of order half the average particle size. The diffusion coefficient $D$ that we obtain from fitting our experimental data (Fig. 3) is ten times larger than this prediction. We notice that, up to now, diffusive behavior has been clearly seen only on experimental systems of no more than ten layers. We must thus remark that our results are also consistent with a crossover to linear broadening at large scales. The shape of the response is better approximated by a Gaussian than by a Lorentzian, as isotropic elasticity would imply. Recent experiments on sand [19, 20] find linear broadening of the response on scales of the order of 100 layers. Similarly in those experiments the precise shape of the response is not Lorentzian. Photoelastic experiments [17, 29] on somewhat smaller piles also suggest linear broadening.

Clearly, larger systems must be studied before stronger conclusions can be drawn from experiments like the one reported in this work. Experiments on more extended systems are under course at present. However in view of the present results, as well as those of previous investigations [16, 17, 19, 20, 29], a possible scenario is to have diffusive behavior at short scales, crossing over to some sort of effective linear elastic behavior (with linear broadening of response) at larger scales. This would be 
in line with the expectation that linear elasticity should be essentially correct at large enough scales.

Our numerical results, on the other hand, show wave-like propagation of stresses, evidenced by two diverging peaks in the response function. There is no sign of crossover to a single-peaked response. This is consistent with previous results [27, 28, 31], and confirms that, on frictionless isostatic systems, disorder does not produce a single-peaked response. Our numerical results also show that the simplifying assumptions leading to the $q$-model are not justifiable for frictionless polydisperse systems.

We remark that our numerical results are valid for the infinitesimal response function, i.e. when contact rearrangements can be ignored [23, 30, 31]. In practice the limit of infinitesimally small perturbation may be very difficult to attain in experiments, so it would be desirable to have a means to quantify the effect of rearrangements in numerical simulations of responses. Recent experiments study the effect of rearrangements in packings subject to relatively large perturbation [43].

On the other hand, in most experimentally feasible se- tups, the existence of friction partially removes the isostaticity properties of granular packings [34]. In the presence of friction the system of equations in terms of interparticle forces becomes indeterminate [32, 33] and accepts a multiplicity of solutions. Thus friction may be seen as an additional source of randomness (apart from geometric and contact disorder). Numerical consideration of friction effects normally makes simulations very time-consuming [32, 33, 44]. Clearly it would be interesting to include the effect of contact rearrangements, and friction, in a realistic but efficient way in simulations.

\section{Acknowledgments}

A.M.V. wishes to thank the Applied Physics Department of CINVESTAV Mérida, where this work was done, for hospitality and support. This work was partially supported by CONACYT, México, through research project 36256-E.
[1] H. Jaeger, S. Nagel, and R. Behringer, Rev. Mod. Phys. 68(4), 1259 (1996).

[2] D. E. Wolf and P. Grassberger, eds., Friction, Arching and Contact Dynamics (World Scientific, Singapore, 1997).

[3] P. de Gennes, Physica A 261(3-4), 267 (1998).

[4] P. de Gennes, Rev. Mod. Phys. 71, S374 (1999), ISSN 0034-6861.

[5] C. Liu, S. Nagel, D. Schecter, S. Coppersmith, S. Majumdar, O. Narayan, and T. Witten, Science 269(5223), 513 (1995).

[6] S. Coppersmith, C. Liu, S. Majumdar, O. Narayan, and T. Witten, Phys. Rev. E 53(5), 4673 (1996).

[7] M. Sexton, J. Socolar, and D. Schaeffer, Phys. Rev. E 60(2), 1999 (1999).

[8] P. Claudin and J. Bouchaud, Phys. Rev. Lett. 78(2), 231 (1997).

[9] J. Bouchaud, M. Cates, and P. Claudin, J. Phys. I 5(6), 639 (1995).

[10] J. Wittmer, P. Claudin, M. Cates, and J. Bouchaud, Nature 382(6589), 336 (1996).

[11] J. Wittmer, M. Cates, and P. Claudin, J. Phys. I 7(1), 39 (1997).

[12] L. Vanel, P. Claudin, J. Bouchaud, M. Cates, E. Clement, and J. Wittmer, Phys. Rev. Lett. 84(7), 1439 (2000).

[13] V. Kenkre, J. Scott, E. Pease, and A. Hurd, Phys. Rev. E 57(5), 5841 (1998).

[14] Kenkre, VM, Granul. Matter 3(1-2), 23 (2001).

[15] J. Bouchaud, P. Claudin, D. Levine, and M. Otto, Eur. Phys. J. E 4(4), 451 (2001).

[16] M. Da Silva and J. Rajchenbach, Nature 406(6797), 708 (2000).

[17] J. Geng, G. Reydellet, E. Clément, and R. P. Behringer, Green's Function measurements of force transmission in 2D granular materials (2003), arXiv:condmat/0211031v3.
[18] J. Scott, V. Kenkre, and A. Hurd, Phys. Rev. E 57(5), 5850 (1998).

[19] G. Reydellet and E. Clement, Phys. Rev. Lett. 86(15), 3308 (2001).

[20] D. Serero, G. Reydellet, P. Claudin, E. Clement, and D. Levine, Eur. Phys. J. E 6(2), 169 (2001).

[21] M. Otto, J. Bouchaud, P. Claudin, and J. Socolar, Phys. Rev. E 67(3), 031302 (2003).

[22] Moukarzel, CF, Phys. Rev. Lett. 81(8), 1634 (1998).

[23] Roux, JN, Phys. Rev. E 61(6), 6802 (2000).

[24] Moukarzel, CF, Granul. Matter 3(1-2), 41 (2001).

[25] Moukarzel, CF, J. Phys.-Condes. Matter 14(9), 2379 (2002).

[26] A. Tkachenko and T. Witten, Phys. Rev. E 60(1), 687 (1999).

[27] A. Tkachenko and T. Witten, Phys. Rev. E 62(2), 2510 (2000).

[28] D. Head, A. Tkachenko, and T. Witten, Eur. Phys. J. E 6(1), 99 (2001).

[29] J. Geng, D. Howell, E. Longhi, R. Behringer, G. Reydellet, L. Vanel, E. Clement, and S. Luding, Phys. Rev. Lett. 8703(3), 5506 (2001).

[30] Roux, JN, Eur. Phys. J. E 7(3), 297 (2002).

[31] D. Head, A. Tkachenko, and T. Witten, Eur. Phys. J. E 7(3), 299 (2002).

[32] F. Radjai, L. Brendel, and S. Roux, Phys. Rev. E 54(1), 861 (1996).

[33] T. Halsey and D. Ertas, Phys. Rev. Lett. 83(24), 5007 (1999).

[34] L. Silbert, D. Ertas, G. Grest, T. Halsey, and D. Levine, Phys. Rev. E 65(3), 031304 (2002).

[35] H. Makse, D. Johnson, and L. Schwartz, Phys. Rev. Lett. 84(18), 4160 (2000).

[36] D. Mueth, H. Jaeger, and S. Nagel, Phys. Rev. E 57(3), 3164 (1998).

[37] D. Blair, N. Mueggenburg, A. Marshall, H. Jaeger, and 
S. Nagel, Phys. Rev. E 6304(4), 1304 (2001).

[38] N. Mueggenburg, H. Jaeger, and S. Nagel, Phys. Rev. E 66(3), 031304 (2002).

[39] G. Lovoll, K. Maloy, and E. Flekkoy, Phys. Rev. E 60(5), 5872 (1999).

[40] A. Vidales, V. Kenkre, and A. Hurd, Granul. Matter 3(12), 141 (2001).

[41] A. Vidales, I. Ippolito, and C. Moukarzel, Physica A 325(3-4), 297 (2003).

[42] P. Claudin, J. Bouchaud, M. Cates, and J. Wittmer,
Phys. Rev. E 57(4), 4441 (1998).

[43] E. Kolb, J. Cviklinski, J. Lanuza, P. Claudin, and E. Clement, Reorganization of a dense granular assembly: the 'unjamming response function' (2003), arXiv:condmat/0308054.

[44] L. Breton, P. Claudin, E. Clement, and J. Zucker, Europhys. Lett. 60(6), 813 (2002).

[45] Similar caveats apply to the results reported in Ref. [16] 